\title{
Safety Assessment of Advanced Imaging Sequences II: Simulations
}

\author{
Jensen, Jørgen Arendt
}

Published in:

IEEE Transactions on Ultrasonics, Ferroelectrics and Frequency Control

Link to article, DOI:

10.1109/TUFFC.2015.2499776

Publication date:

2016

Document Version

Peer reviewed version

Link back to DTU Orbit

Citation (APA):

Jensen, J. A. (2016). Safety Assessment of Advanced Imaging Sequences II: Simulations. IEEE Transactions on Ultrasonics, Ferroelectrics and Frequency Control, 63(1), 120 - 127.

https://doi.org/10.1109/TUFFC.2015.2499776

\section{General rights}

Copyright and moral rights for the publications made accessible in the public portal are retained by the authors and/or other copyright owners and it is a condition of accessing publications that users recognise and abide by the legal requirements associated with these rights.

- Users may download and print one copy of any publication from the public portal for the purpose of private study or research.

- You may not further distribute the material or use it for any profit-making activity or commercial gain

- You may freely distribute the URL identifying the publication in the public portal

If you believe that this document breaches copyright please contact us providing details, and we will remove access to the work immediately and investigate your claim. 


\title{
Safety Assessment of Advanced Imaging Sequences II: Simulations
}

\author{
Jørgen Arendt Jensen, IEEE Fellow \\ Center for Fast Ultrasound Imaging, Department of Electrical Engineering, \\ Technical University of Denmark, DK-2800 Lyngby, Denmark
}

\begin{abstract}
An automatic approach for simulating the emitted pressure, intensity, and MI of advanced ultrasound imaging sequences is presented. It is based on a linear simulation of pressure fields using Field II, and it is hypothesized that linear simulation can attain the needed accuracy for predicting Mechanical Index (MI) and $I_{\text {spta. } 3}$ as required by FDA. The method is performed on four different imaging schemes and compared to measurements conducted using the SARUS experimental scanner. The sequences include focused emissions with an F-number of 2 with 64 elements that generate highly non-linear fields. The simulation time is between $0.67 \mathrm{~ms}$ to $2.8 \mathrm{~ms}$ per emission and imaging point, making it possible to simulate even complex emission sequences in less than $1 \mathrm{~s}$ for a single spatial position. The linear simulations yield a relative accuracy on MI between $-12.1 \%$ to $52.3 \%$ and for $I_{s p t a .3}$ between $\mathbf{- 3 8 . 6 \%}$ to $62.6 \%$, when using the impulse response of the probe estimated from an independent measurement. The accuracy is increased to between $-22 \%$ to $24.5 \%$ for MI and between $-33.2 \%$ to $27.0 \%$ for $I_{s p t a .3}$, when using the pressure response measured at a single point to scale the simulation. The spatial distribution of MI and $I_{t a .3}$ closely matches that for the measurement, and simulations can therefore be used to select the region for measuring the intensities, resulting in a significant reduction in measurement time. It can validate emission sequences by showing symmetry of emitted pressure fields, focal position, and intensity distribution.
\end{abstract}

\section{INTRODUCTION}

An efficient method for measuring intensities and Mechanical Index (MI) for complex imaging sequences has been presented in the accompanying paper [1]. The approach was implemented on the SARUS (Synthetic Aperture Real-time Ultrasound System) experimental ultrasound scanner [2], and uses one receive channel in the scanner to acquire the hydrophone signal. Intensity mapping of the full sequence is conducted in 1 to 2 hours, rather than the days currently needed for this. It is argued that the approach is less error prone and more accurate than the more cumbersome and manual approaches using an oscilloscope.

The method, thus, solves some of the inherent problems in measuring on complex ultrasound sequences, but it must still be ensured that the measurements are conducted at the correct spatial positions. If the measured values are above the limits, the sequence must be adjusted and measured again, which is cumbersome. Daft et al. [3] therefore suggested using simulation to cut down on measurement time. They used a rather complicated non-linear simulation method. This paper suggests using linear Field II [4], [5] simulations prior to measurements to ease the sequence planning and reduce measurement time. The hypothesis is that the simulation accuracy can be within the $30 \%$ accuracy allowed by the FDA, even though a linear simulation method is employed. This results in a very fast simulation times of minutes, which is usable for experimentation and designing sequences.

The approach relies on automatic decoding of the imaging sequence and a calibration step for the transducer's impulse response as described in Section II. Four different imaging sequences are simulated for convex and linear array probes and compared to the corresponding measurements to investigate the accuracy in Section IV.

The method also makes it possible to test the imaging schemes. The automatic decoding of sequences facilitates a fully automated simulation of the emitted field. It can then be checked for symmetry, focus position, emission order, and intensity distribution as described in Section V.

\section{LINEAR SIMULATION OF INTENSITY LEVELS}

Both $I_{t a}$ and MI are derived from the emitted pressure, which in Field II is modeled as [6], [7]:

$$
p(\vec{r}, t)=\rho \frac{\partial v_{\text {imp }}(t)}{\partial t} * h(\vec{r}, t) * e(t),
$$

where $t$ is time, $\vec{r}$ is position of the point, $h(\vec{r}, t)$ is the spatial impulse response, and $\partial v_{\text {imp }}(t) / \partial t$ is the probe impulse response. This is the impulse response from the voltage applied onto the transducer terminals to the surface acceleration, assuming that the transducer element vibrates as a piston. The last term $e(t)$ is the electrical excitation of the probe, which is usually a single cycle sinusoid for B-mode imaging and several cycles for flow imaging. Assuming a linear medium, the emitted field from a multi element probe with $N_{e}$ elements is

$$
\begin{aligned}
p(\vec{r}, t) & =\rho \frac{\partial v_{\text {imp }}(t)}{\partial t} * e(t) * \sum_{i=1}^{N_{e}} a_{i} h_{i}\left(\vec{r}, t-\tau_{i}\right) \\
& =\rho v_{e}(t) * e(t) * \sum_{i=1}^{N_{e}} a_{i} h_{i}\left(\vec{r}, t-\tau_{i}\right) \\
v_{e}(t) & =\frac{\partial v_{\text {imp }}(t)}{\partial t} .
\end{aligned}
$$

Here $a_{i}$ is the apodization or weight on element $i$ and $\tau_{i}$ is the time delay for focusing. Field II employs this model for calculating the emitted pressure field and non-linear propagation is neglected [4], [5]. It is assumed that all elements vibrate as pistons, and that the excitation and impulse responses can be 
assumed to be the same across all elements. This is often a valid assumption for commercial, high quality probes.

The Field II simulation model must be calibrated to reveal the correct pressure levels. Therefore the sampled version of $v_{e}(t)$ equal to $v_{e}(n)$ should be determined.

\section{A. Determination of transducer impulse response}

The approach here is to determine the impulse response of the probe. It can be found from modeling the probe material as described in [8], [9], [10], [11]. This entails knowing all the material parameters for the probe along with the matching layers. There are usually many parameters and layers, and it is often impossible to know all with a sufficient precision, or they are company secrets. It is therefore chosen here to estimate the impulse response. For a single, small element at the elevation focus of the array $h(\vec{r}, t)$ approaches a Dirac delta function. Exciting the probe with a Gaussian, random signal $e_{r}(n)$ and measuring the emitted pressure at the elevation focus then yields the impulse response through a cross-correlation of the excitation signal with the received pressure signal. For a discrete signal the cross-correlation is calculated as:

$$
\begin{aligned}
R_{e p}(k) & =\frac{1}{N} \sum_{n=1}^{N} e_{r}(n) p(\vec{r}, n+k) \\
& =\frac{1}{N} \sum_{n=1}^{N} e_{r}(n) \rho e_{r}(n+k) * v_{e}(n+k) * h(\vec{r}, n+k) \\
& =\rho R_{e}(k) * v_{e}(k) * h(\vec{r}, k)
\end{aligned}
$$

where $N$ is the length of the receive signal. Assuming that $R_{e}(k)$ is white, and that the transfer function of $h(\vec{r}, k)$ has a constant gain $k_{h}$ in the relevant bandwidth of the transducer gives:

$$
R_{e p}(k)=\rho P_{e} k_{h} v_{e}(k),
$$

where $P_{e}$ is the power of the random excitation signal $\left(R_{e}(k)=\right.$ $\left.P_{e} \delta(k)\right)$. The impulse response can, thus, be found as:

$$
v_{e}(k)=\frac{R_{e p}(k)}{\rho P_{e} k_{h}} .
$$

The constant $k_{h}$ can be found from the Fourier transform of $h(\vec{r}, k)$ at the center frequency $f_{0}$ :

$$
k_{h}=\left|\sum_{n=1}^{N} h(\vec{r}, n) \exp \left(-j 2 \pi f_{0} \frac{n}{f_{s}}\right)\right|,
$$

where $f_{s}$ is the sampling frequency. $k_{h}$ can also be found from an average in the relevant band. Another approach is to deconvolve the spatial impulse response to reveal the impulse response as in [12].

The scaled impulse response to use in Field II $v_{e I I}(k)$ is then given by

$$
v_{e I I}(k)=\rho v_{e}(k) f_{s}^{2}=\frac{R_{e p}(k) f_{s}^{2}}{P_{e} k_{h}},
$$

as the convolution operation in Field II includes a division with the sampling frequency for each convolution to yield results independent of a change in sampling frequency.

\section{B. Compensation through scaling}

Non-linear effects in the water will distort the propagating wave and the linear simulation will not match the shape of the non-linear wave. There is, however, little absorption of energy in water, and a possible solution is to scale the wave based on the energy in the responses with a scaling factor of:

$$
k_{v_{e}}=\sqrt{\frac{\sum_{n=1}^{N} p_{s}^{2}(\vec{r}, n)}{\sum_{n=1}^{N} p_{m}^{2}(\vec{r}, n)}},
$$

where $p_{m}(\vec{r}, n)$ is the measured pressure and $p_{s}(\vec{r}, n)$ the simulated. The impulse response used in the simulation is then:

$$
v_{e s}(n)=k_{v_{e}} v_{e I I}(n) .
$$

This scaling is also investigated in the simulation scripts.

\section{Measurement SetuP}

The intensities were measured with the setup described in the accompanying paper [1]. The SARUS system [2] was used for generating the emissions for acquiring the hydrophone signal. An Onda AIMS III system (Onda Corporation, Sunnyvale, CA, USA) was controlled through Matlab (MathWorks, Natick, MA, USA) for moving the hydrophone around in the scan region. The SARUS scanner stores a parameter file after each scan, where the file describes the complete setup and scan sequence executed.

\section{A. Ultrasound sequences}

Four imaging sequences have been used for the experiments. The first is a simple fixed focus sequence with a focus at $40 \mathrm{~mm}$, and the beam direction is along the center axis of the transducer. The second sequence makes a simple duplex scan, where a 129 line B-mode sequence is interleaved with 129 flow emissions [13], [14]. The third example is a vector flow imaging scheme (VFI sequence) in 17 directions with 16 emissions each followed by a 129 lines B-mode image [15]. The fourth is a duplex Synthetic Aperture (SA) sequence with 7 flow emissions inter-spaced with $129 \mathrm{~B}$-mode emissions suitable for high dynamic range flow imaging [16]. All four sequences along with the ultrasound probes used are described in detail in the accompanying paper [1].

\section{RESULTS FROM SIMULATIONS OF SEQUENCES}

In this Section the four measured sequences are simulated using Field II. The set-up of the simulation is made automatically by reading the parameter file stored by the SARUS scanner. It defines the transducer, scanning sequence's focusing, and apodization for all emissions. The simulations are conducted at the same points as measured, and the same type of automatic report as for the measurements is generated [1]. The user, thus, only needs to give the name of the measurement's parameter file to make the simulation. 

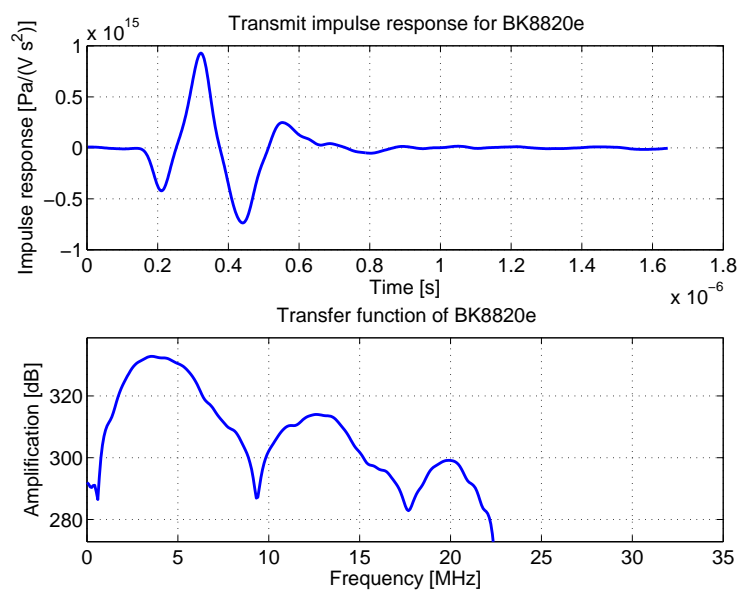

Fig. 1. Estimated impulse response of the BK Medical 8820e convex array probe (top graph) along with the corresponding transfer function.

\section{A. Calibration of simulation}

The impulse response of the BK Medical 8820e convex array probe has been identified using coded sequences as described in Section II-A. The signal at the elevation focus of the probe was measured using 10 coded signals with a length of $10 \mu \mathrm{s}$, where only the central element of the probe was used for emission to ensure linear propagation. The hydrophone was placed at the elevation focus depth and in front of the element's center. The resulting impulse response is shown in Fig. 1. This has been used as the impulse response of the probe in the simulations.

The excitation signal in the sequence is used multiplied with the proper excitation voltage of the transmitter. This method makes it possible to predict intensities and MI without prior measurement on the sequence, and only necessitates one calibration measurement of the impulse response for each probe. The same single element has been used with a one cycle excitation and the impulse response has been used in modeling the response. This is shown in Fig. 2, where the solid line is the measured pressure wave and the dashed line is the simulated response. The relative RMS error between the two response are calculated as:

$$
R M S_{\text {rel }}=\sqrt{\frac{\sum_{n=1}^{N}\left(p_{m}(\vec{r}, n)-p_{s}(\vec{r}, n)\right)^{2}}{\sum_{n=1}^{N} p_{m}^{2}(\vec{r}, n)}} .
$$

It is $20.9 \%$, and all the major oscillations in the response are captured in the simulated result.

The impulse response has also been measured for the BK Medical 8670 linear array probe and is shown in Fig. 3. This shows the wider bandwidth and higher center frequency of the linear array probe. A measurement with a single cycle $8 \mathrm{MHz}$ excitation is show as the blue solid curve in the lower graph. A simulation of this using the estimated impulse response is shown as the dashed red curve. The simulation captures all major oscillations in the response and the magnitude has the correct order. The relative RMS error is $29.4 \%$.

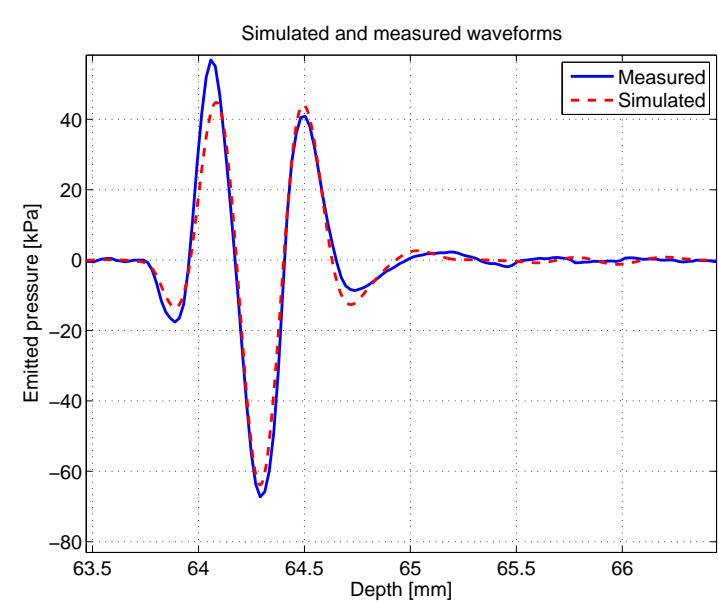

Fig. 2. Measured and simulated pressure responses of the BK Medical 8820e convex array probe.
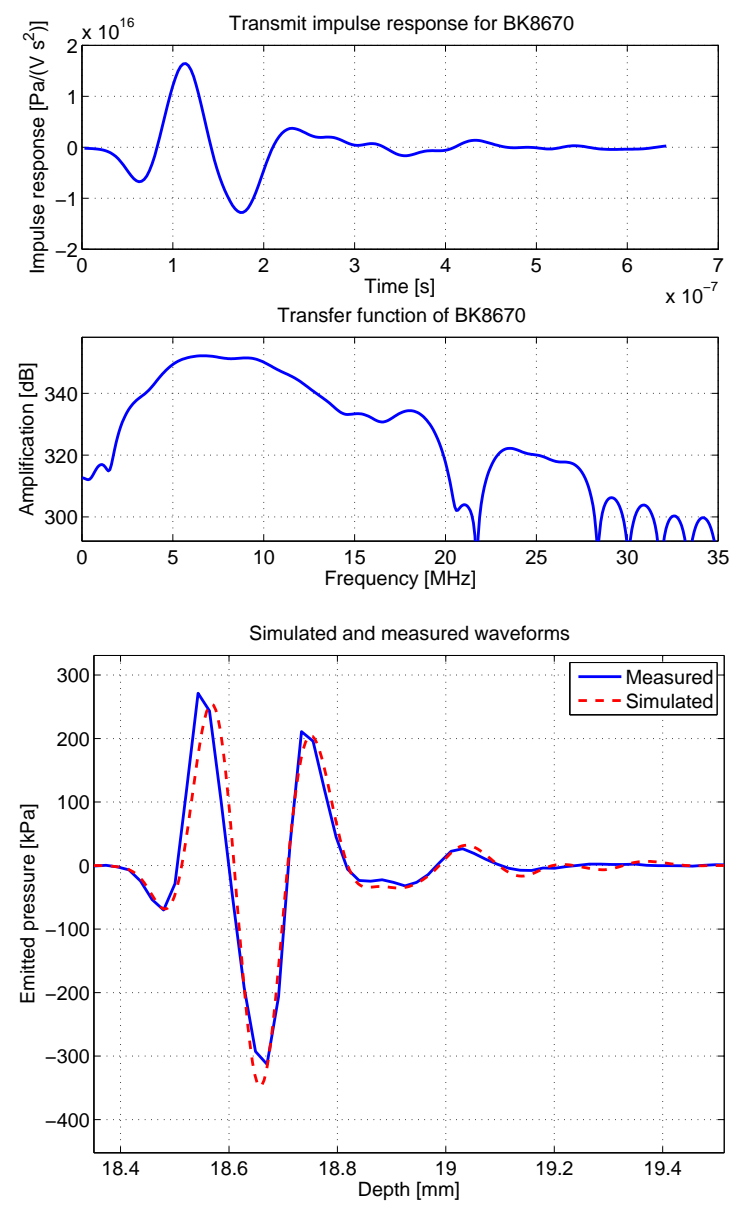

Fig. 3. Estimated impulse response of the BK Medical 8670 linear array probe (top graph) along with the corresponding transfer function. The bottom graph shows the simulated response, when using this impulse response and a single cycle $8 \mathrm{MHz}$ excitation pulse. The solid blue curve shows the corresponding measured response. 


\section{B. Simulation results}

The results from simulating the single focus sequence using the impulse response is shown in Fig. 4 and should be compared to the corresponding Fig. 5 in the accompanying paper. For the duplex sequence it is Fig. 5, which should be compared with Fig. 7 in the measurement paper. For VFI Fig. 6 should be compared to Fig. 8 .

Table I lists the measured and simulated derated MI and $I_{\text {spta.3 }}$ for all four imaging examples for both the direct impulse response method and a scaled simulation. The fourth columns holds the simulated values, when using the measured impulse response directly. In the fifth column a single measurement point has been used for scaling the impulse response by a factor based on the ratio between the energy of the measured and simulated response. Columns 6 and 7 show the accuracy of the two simulations relative to the measured value. The relative accuracy spans from -12.1 to $52.3 \%$ for MI. For $I_{\text {spta.3 }}$ the relative accuracy is from $-38.6 \%$ to $62.6 \%$, which is above the required FDA accuracy of $30 \%$. MI is slightly harder to predict due to the influence from non-linear propagation effects, but simulation can still give an early and realistic indication whether a sequence is roughly within FDA limits. Using the calibration measurement improves on the accuracy, as the non-linear effect is partly taken into account. The relative accuracy then spans from -22 to $24.5 \%$ for MI with a mean value of $9.3 \%$. For $I_{\text {spta.3 }}$ the relative accuracy is now from $-33.16 \%$ to $27.0 \%$ with a mean value of $-10.2 \%$. The simulation can therefore in all cases apart from one give an accuracy within the FDA requirement of $30 \%$

For the single emission sequence the peak value is predicted to be at $(0,0,42) \mathrm{mm}$, where the intensity measurement gives $(0,0,41) \mathrm{mm}$. For $\mathrm{MI}$ it is $(0,0,42) \mathrm{mm}$ and $(0,0,37) \mathrm{mm}$. This is also the easiest and most predictable sequence for the spatial peak values, as it has one unique maximum. The VFI sequence has an intensity peak value at $(2,0,20) \mathrm{mm}$, where it is simulated to be at $(14,0,40) \mathrm{mm}$ due to a fairly uniform intensity field in the primary imaging area. The MI position is predicted to be at $(30,0,40) \mathrm{mm}$ and measured to $(8$, $0,40) \mathrm{mm}$. This is at the B-mode transmit focus, which is at the same depth for the different lateral positions. Small variations in the transducer element sensitivity can therefore give a lateral peak position at any of the emitted directions. The same phenomenon is seen for the duplex sequence in Fig. 5, where MI again is dominated by the B-mode focused emission. The intensity is fairly uniform in the central imaging area and has a standard deviation of less than $1.2 \mathrm{~dB}$ in the lateral direction and the largest change is $4.9 \mathrm{~dB}$ from the highest to the lowest value. A $1.2 \mathrm{~dB}$ change is a $15 \%$ change and $4.9 \mathrm{~dB}$ corresponds to a $75 \%$ change in intensity value.

\section{Simulation times}

For the fixed focus emission sequence the whole simulation takes $2.76 \mathrm{~s}$ using the Field IIpro version 4.61, 28/6-2015 [17] on a Dual Intel Xeon E5-2630 2.60 GHz CPU with 32 Gbytes RAM under Ubuntu Linux 12.04LTS and Matlab version 2013b. Here one emission is made and 1,001 points are simulated, so that the time for each point is $2.8 \mathrm{~ms}$.

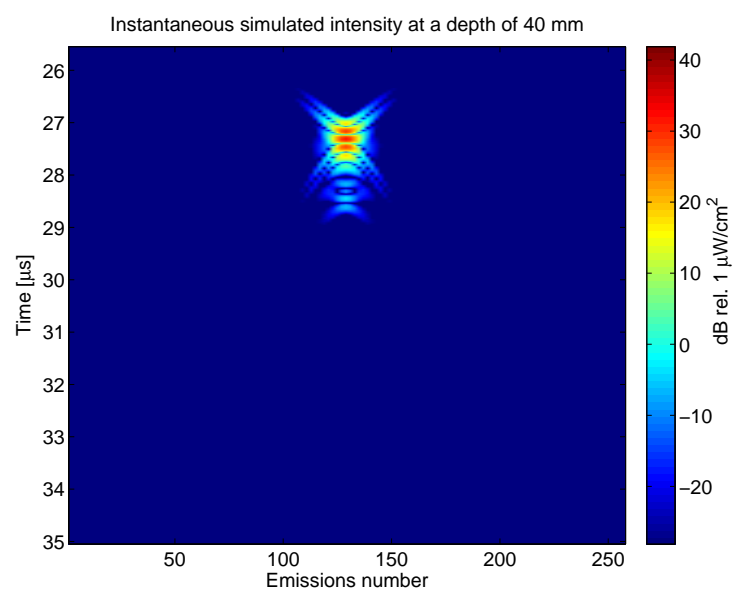

Fig. 8. Distribution of the instantaneous intensity as a function of time and emission number for a depth of $40 \mathrm{~mm}$ for the B-mode imaging pulse without quantization of the transmit delays. The color values are shown in $\mathrm{dB}$ relative to $1 \mu \mathrm{W} / \mathrm{cm}^{2}$

For the duplex sequence 2,121 points are simulated with 258 emissions per point, resulting in 547,218 emissions. The time for this is $364.4 \mathrm{~s}$ giving a simulation time of $0.67 \mathrm{~ms}$ for each emission. The VFI sequence has 533 points with 673 emissions each. The time for this is 272.2 s, giving a simulation time of $0.76 \mathrm{~ms}$ for each emission.

\section{VALIDATION OF IMAGING SEQUENCES}

The acquired data can also be used to validate the emitted pressure field, and that the emission sequence is correct. This can be performed by plotting the emitted pressure signal as a function of emission number or as a function of spatial position for a fixed emission. The first method gives an indication of the symmetry of emitting the beams. The second approach displays the spread of the transmitted field. Comparing these measured responses with the simulated responses gives an indication of the quality of the probe and the transmit amplifiers in terms of uniformity and missing elements or transmitters.

An example of the first type plot is shown in Fig. 7 for only B-mode emissions in the VFI sequence at a hydrophone position of $(0,0,40) \mathrm{mm}$. The symmetry of the response indicates that all elements and all transmitters are working. The corresponding simulation is shown on the right graph. There are fairly high far side-lobes, which are due to the quantization of the transmit delays. The field without transmit quantization is shown in Fig. 8. This shows how the measurement and simulation approach can be used to investigate the quality of the transmitted field and its optimization.

The distribution of intensities for the whole duplex sequence is shown in Fig. 9. There is a significant contribution for every second emission from the flow emission. This plot can be used to ensure the correctness of the scan sequence.

The spread of the emitted pressure field can also be studied by combining measurements from a number of hydrophone positions. This is shown again for the VFI simulation for both the measured data on the left and the corresponding simulation on the right plot in Fig. 10. Both yield roughly the 

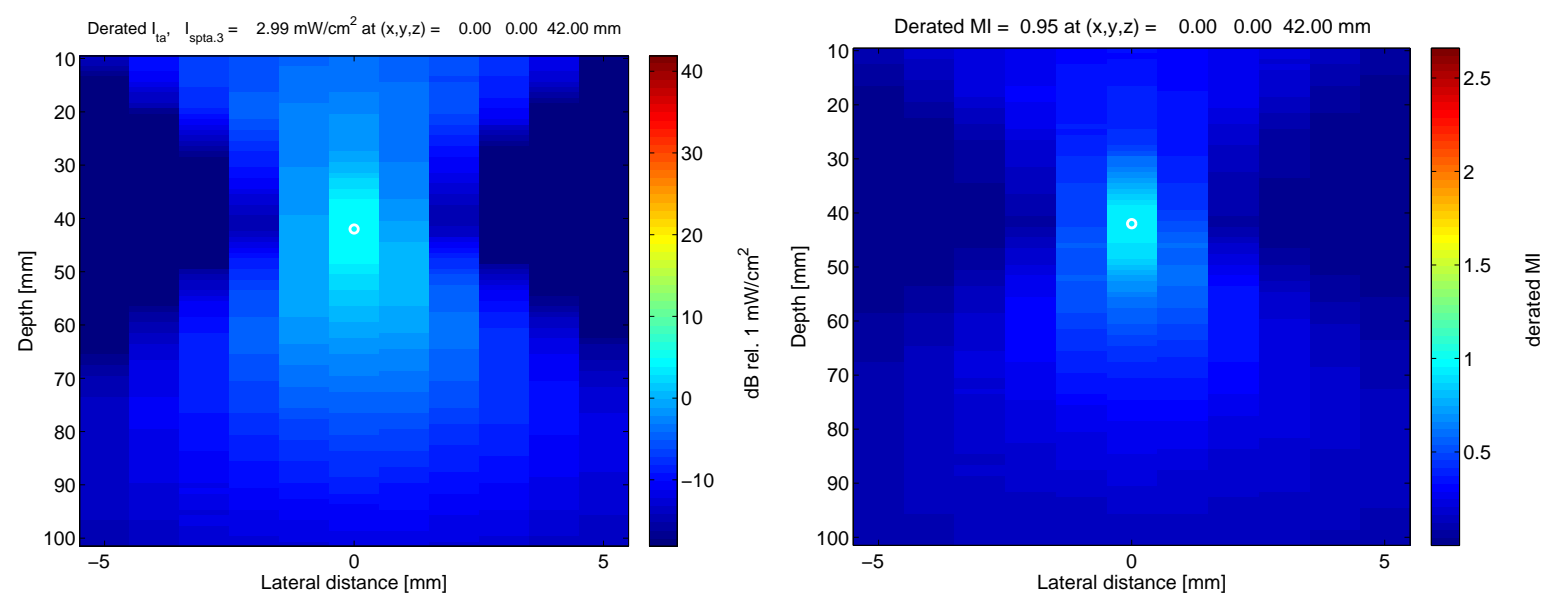

Fig. 4. Simulated results for the single emission sequence as a function of spatial position. The derated temporal averaged intensity is shown in the left image and the derated MI is shown on the right. The white circle indicates the position of the peak values.
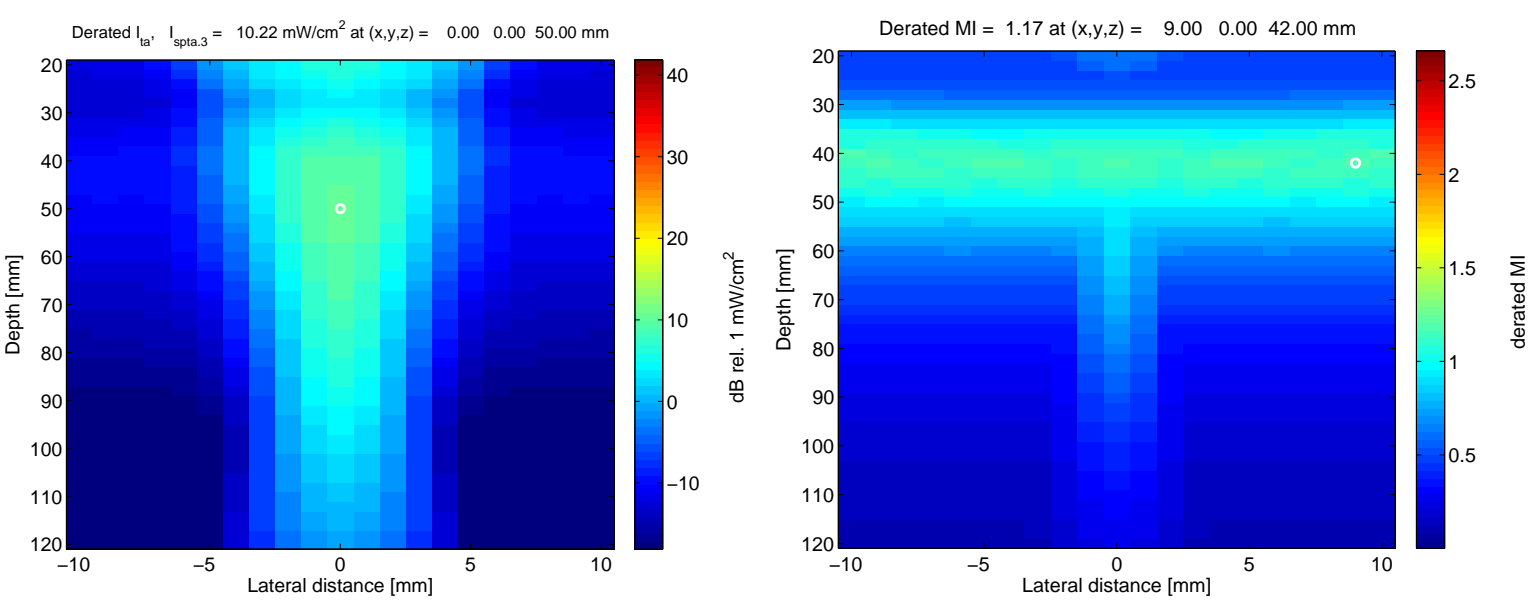

Fig. 5. Simulated results for the duplex B-mode and flow sequence as a function of spatial position. The derated temporal averaged intensity is shown in the left image and the derated MI is shown on the right. The white circle indicates the position of the peak values.
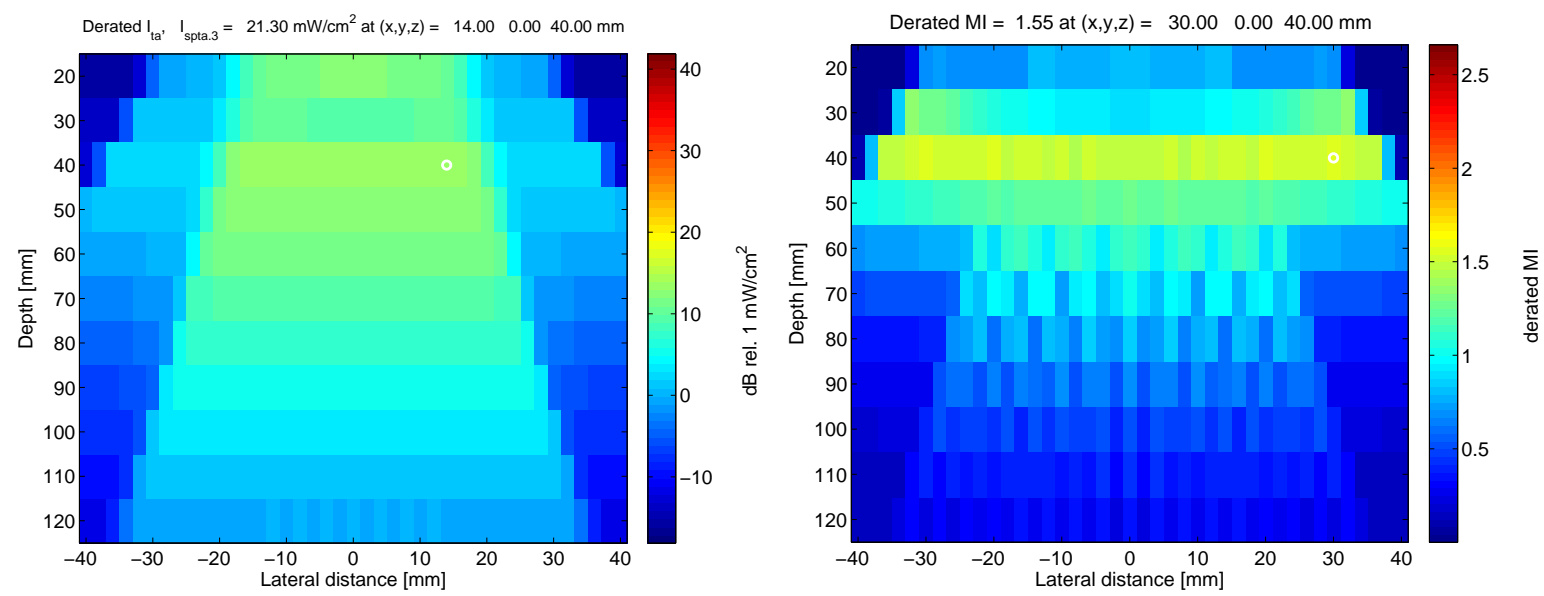

Fig. 6. Simulated results for the VFI sequence as a function of spatial position. The derated temporal averaged intensity is shown in the left image and the derated MI is shown on the right. The white circle indicates the position of the peak values. 
This is the author's version of an article that has been published in this journal. Changes were made to this version by the publisher prior to publication.

The final version of record is available at http://dx.doi.org/10.1109/TUFFC.2015.2499776

TABLE I

MEASURED AND SIMUlATED MI AND $I_{\text {spta.3 }}$ FOR ALL FOUR IMAGING EXAMPLES ALONG WITH THE RELATIVE ACCURACY. THE HEADING DESCRIBES THE CONTENT OF THE COLUMNS. THE THIRD COLUMN CONTAINS THE MEASURED VALUES, AND THE FOURTH COLUMN "MEASURED IMPULSE" HOLDS THE SIMULATED VALUES, WHEN USING THE MEASURED IMPULSE RESPONSE. A SCALING A HAS BEEN APPLIED ON THIS FOR THE FIFTH COLUMN. THE RELATIVE ACCURACY IN REFERENCE TO THE MEASURED VALUES ARE SHOWN IN COLUMNS 6 AND 7.

\begin{tabular}{|l|c|r|r|r|r|r|c|}
\hline $\begin{array}{l}\text { Imaging } \\
\text { sequence }\end{array}$ & Parameter & $\begin{array}{r}\text { Measured } \\
\text { value }\end{array}$ & $\begin{array}{r}\text { Measured } \\
\text { Impulse }\end{array}$ & $\begin{array}{c}\text { Simulated } \\
\text { Scaling. }\end{array}$ & $\begin{array}{c}\text { Rel. acc. } \\
\text { Impulse }\end{array}$ & $\begin{array}{c}\text { Rel. acc. } \\
\text { Scaling }\end{array}$ & Unit \\
\hline \hline Single emission & MI & 0.81 & 1.11 & 0.95 & $37.0 \%$ & $17.3 \%$ & \\
& $I_{\text {spta.3 }}$ & 3.28 & 4.06 & 2.99 & $23.8 \%$ & $-6.4 \%$ & $\mathrm{~mW} / \mathrm{cm}^{2}$ \\
\hline Duplex flow & MI & 0.94 & 1.00 & 1.17 & $6.0 \%$ & $24.5 \%$ & \\
& $I_{\text {spta.3 }}$ & 14.42 & 8.85 & 10.22 & $-38.6 \%$ & $-29.1 \%$ & $\mathrm{~mW} / \mathrm{cm}^{2}$ \\
\hline VFI & MI & 1.32 & 2.01 & 1.55 & $52.3 \%$ & $17.4 \%$ & \\
& $I_{\text {spta.3 }}$ & 31.87 & 38.83 & 21.30 & $21.8 \%$ & $-33.2 \%$ & $\mathrm{~mW} / \mathrm{cm}^{2}$ \\
\hline SA & MI & 0.91 & 0.80 & 0.71 & $-12.1 \%$ & $-22.0 \%$ & \\
& $I_{\text {spta.3 }}$ & 4.81 & 7.82 & 6.11 & $62.6 \%$ & $27.0 \%$ & $\mathrm{~mW} / \mathrm{cm}^{2}$ \\
\hline
\end{tabular}
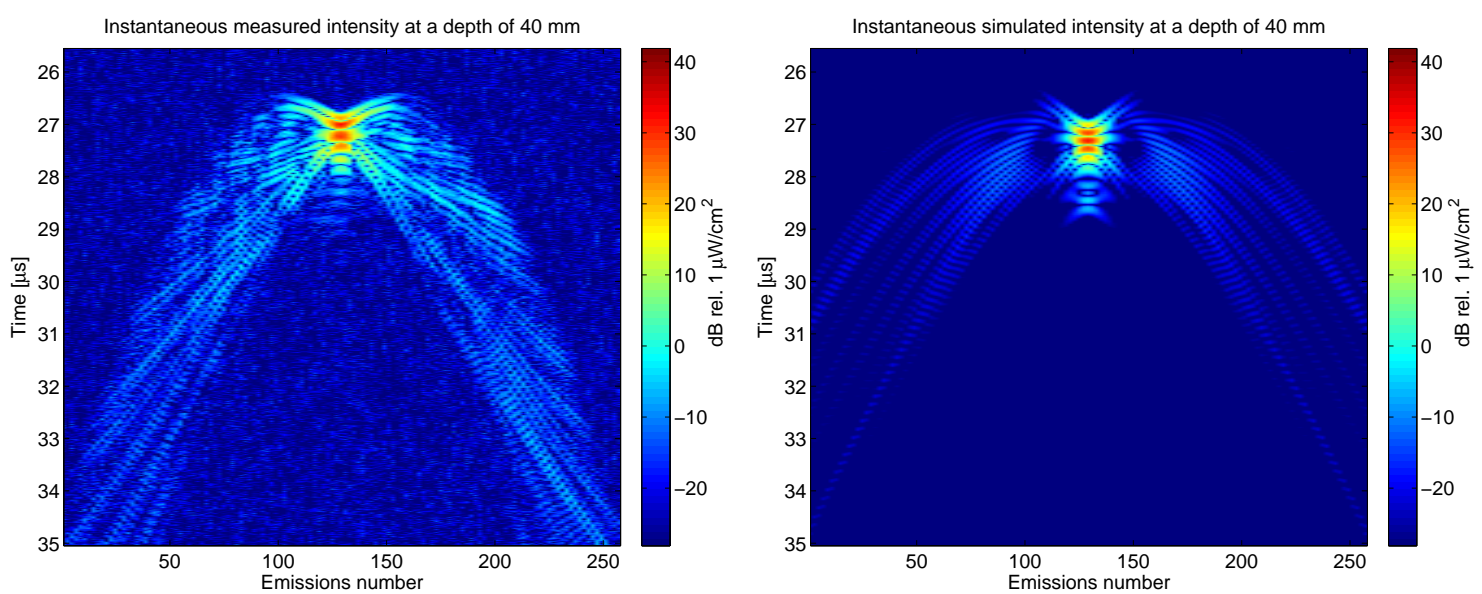

Fig. 7. Distribution of the instantaneous intensity as a function of time and emission number for a depth of 40 mm for the B-mode imaging pulse.The color values are shown in $\mathrm{dB}$ relative to $1 \mu \mathrm{W} / \mathrm{cm}^{2}$. The left graph shows the measured data and the right shows the simulated field.
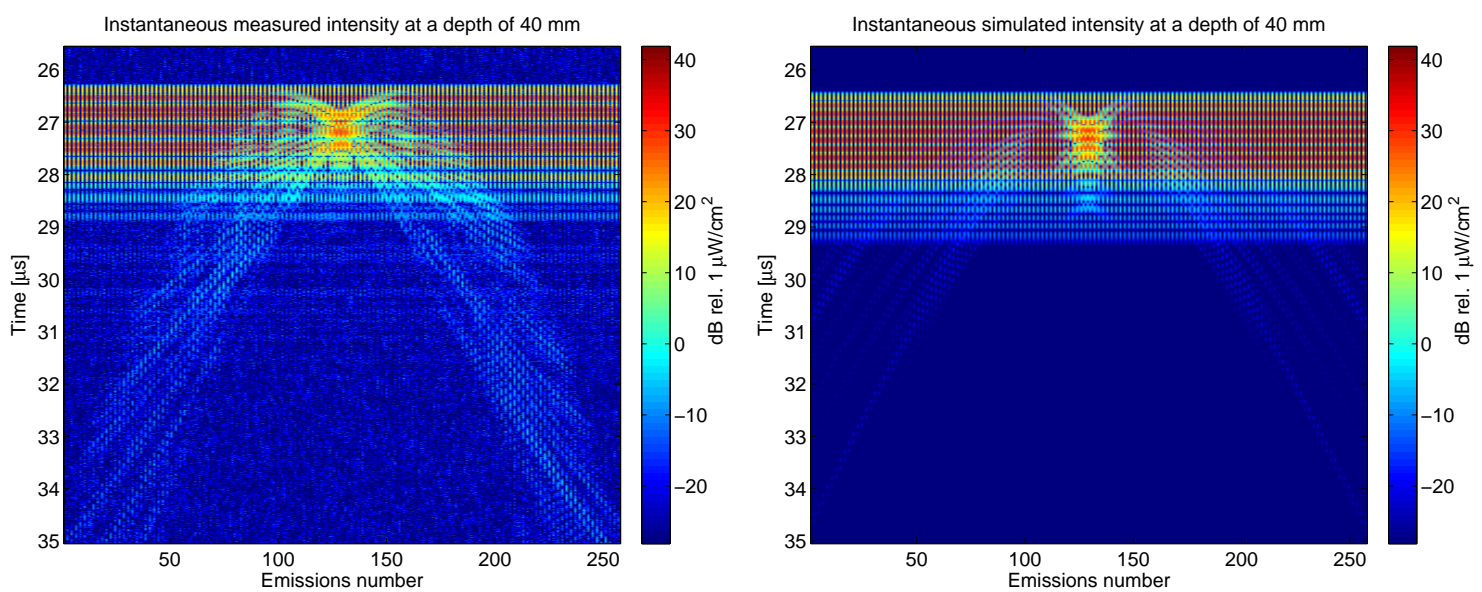

Fig. 9. Distribution of the instantaneous intensity as a function of time and emission number for a depth of $40 \mathrm{~mm}$ for the duplex imaging sequence. The color values are shown in $\mathrm{dB}$ relative to $1 \mu \mathrm{W} / \mathrm{cm}^{2}$, where the left graph shows the measured data and the right is simulated. 
same width and the effect of a non-ideal transducer and nonlinear propagation can be seen from the difference between the plots. The quality of the alignment can also be estimated from this figure, when assuming a symmetric response. The 6-dB contours reveals an off-axis center position of $79.7 \mu \mathrm{m}$ or 0.18 $\lambda$. This corresponds to a roughly $3.5 \%$ drop in amplitude.

\section{DISCUSSION}

A method for the simulation of advanced imaging sequence has been presented. In many sequences the important area of the peak intensity and MI is confined to a smaller region. This can be uncovered using simulations as show in Section IV.

Although a linear simulation model is used, it is possible to predict the position of the derated values for both $I_{\text {spta.3 }}$ and MI, and how closely the spatial sampling should be performed. This also makes it possible to rapidly prototype new sequences, and validate the possibility for implementation using simulation based on only having a measured impulse response for the probe used.

The simulations yield results within $\pm 25 \%$ for MI and from $-33.16 \%$ to $27.0 \%$ for $I_{\text {spta. } 3}$, when using the measured and scaled transducer impulse response of the probe. This accuracy is very close to the FDA requirements of $30 \%$ and can give an accurate early indication of the level and spatial positions of MI and $I_{\text {spta.3. }}$. The simulation is linear and completely neglects non-linear effects. These was partly compensated for by scaling the simulations according to the energy at a single point. This improves on the accuracy to be nearly within FDA requirements.

Several things affects the accuracy of both simulations and measurements. The measurements have an uncertainty from the alignment of the probe to the hydrophone. A misalignment within 1 to 2 wavelengths around 0.5 to $1 \mathrm{~mm}$ can make the signal drop by $6 \mathrm{~dB}(50 \%)$. From the symmetry of the measured PSF in Fig. 10, the alignment on the center axis is estimated to be $79.7 \mu \mathrm{m}$, which results in an uncertainty in intensity measurements about $3.5 \%$. The probe model also affects the accuracy of the simulation. This includes the exact physical layout of the probe including the kerf, the boundary conditions of the simulation, whether the elements are vibrating as pistons, and that all elements are vibrating with the same amplitude.

Ideally, non-linear effects should be taken into account, but this will dramatically increase simulation time as indicated in the literature [18], [19]. Actually, the simulation times then become larger than the time needed for a complete measurement, which defies the purpose of making simulations. The simulations conducted here take around 0.67 to $2.8 \mathrm{~ms}$ per emission and point, and a complete sequence can be simulated in a few minutes. This makes it possible to make easy experimentations with different allocations of the intensity budget.

Both the measurements and simulations offer the possibility for validating the transmitted pressure field as both automatically yield the point spread function for the emitted field. It can then easily be checked for symmetry, and that the focus peaks at the correct depth and spatial position.

\section{CONCLUSIONS AND RECOMMENDATIONS}

Simulations and measurements of advanced imaging sequences have been compared and shown to reveal similar results nearly within the FDA rule of $30 \%$ accuracy. The results are within what can be expected from the inaccuracy in transducer alignment and modeling errors in the simulation. Adequate agreement is, thus, found even though strong nonlinear effects can be observed in the measurements, which are not taken into account in the linear simulation. This is due to the low absorption of water and the preservation of energy in the simulated responses. The Field II model can, thus, predict intensity levels and MI to a high degree, and is both an efficient and fast method for evaluating new imaging schemes before they are implemented, and for guiding the actual safety measurements.

\section{ACKNOWLEDGEMENT}

This work was supported by grant grant 82-2012-4 from the Danish Advanced Technology Foundation and by BK Ultrasound.

\section{REFERENCES}

[1] J. A. Jensen, M. F. Rasmussen, M. J. Pihl, S. Holbek, C. A. VillagomezHoyos, D. P. Bradway, M. B. Stuart, and B. G. Tomov, "Safety assessment of advanced imaging sequences, I: Measurements," IEEE Trans. Ultrason., Ferroelec., Freq. Contr. , p. Submitted, 2015.

[2] J. A. Jensen, H. Holten-Lund, R. T. Nilsson, M. Hansen, U. D. Larsen, R. P. Domsten, B. G. Tomov, M. B. Stuart, S. I. Nikolov, M. J. Pihl, Y. Du, J. H. Rasmussen, and M. F. Rasmussen, "SARUS: A synthetic aperture real-time ultrasound system," IEEE Trans. Ultrason., Ferroelec., Freq. Contr. 60(9), pp. 1838-1852, 2013.

[3] C. M. W. Daft, W. M. Leue, K. E. Thomenius, L. A. Odegaard, M. C. MacDonald, and A. S. Meyers, "Acoustic intensity simulations for regulatory compliance," Proceedings of the SPIE - the International Society for Optical Engineering 3664, pp. 212-220, 1999.

[4] J. A. Jensen and N. B. Svendsen, "Calculation of pressure fields from arbitrarily shaped, apodized, and excited ultrasound transducers," IEEE Trans. Ultrason., Ferroelec., Freq. Contr. 39, pp. 262-267, 1992.

[5] J. A. Jensen, "Field: A program for simulating ultrasound systems," Med. Biol. Eng. Comp. 10th Nordic-Baltic Conference on Biomedical Imaging, Vol. 4, Supplement 1, Part 1, pp. 351-353, 1996.

[6] G. E. Tupholme, "Generation of acoustic pulses by baffled plane pistons," Mathematika 16, pp. 209-224, 1969.

[7] P. R. Stepanishen, "Transient radiation from pistons in an infinite planar baffle," J. Acoust. Soc. Am. 49, pp. 1629-1638, 1971.

[8] W. P. Mason, Electromechanical transducers and wave filters, D. Van Nostrand Co., 1942.

[9] E. Sittig, "Transmission parameters of thickness-driven piezoelectric transducers arranged in multilayer configurations," IEEE Transactions on Sonics and Ultrasonics 14(4), pp. 167-174, 1967.

[10] P. Marechal, F. Levassort, L.-P. Tran-Huu-Hue, and M. Lethiecq, "Lensfocused transducer modeling using an extended KLM model," Ultrasonics 46(2), pp. 155-167, 2007.

[11] D. Bæk, J. A. Jensen, and M. Willatzen, "Modeling transducer impulse responses for predicting calibrated pressure pulses with Field II," $J$. Acoust. Soc. Am. 127, pp. 2825-2835, May 2010.

[12] K. A. Wear, P. M. Gammell, S. Maruvada, Y. Liu, and G. R. Harris, "Improved measurement of acoustic output using complex deconvolution of hydrophone sensitivity," IEEE Trans. Ultrason., Ferroelec., Freq. Contr. 61(1), pp. 62-75, 2014.

[13] D. H. Evans and W. N. McDicken, Doppler Ultrasound, Physics, Instrumentation, and Signal Processing, John Wiley \& Sons, New York, 2000.

[14] J. A. Jensen, Estimation of Blood Velocities Using Ultrasound: A Signal Processing Approach, Cambridge University Press, New York, 1996.

[15] J. A. Jensen, A. H. Brandt, and M. B. Nielsen, "Convex array vector velocity imaging using transverse oscillation and its optimization," IEEE Trans. Ultrason., Ferroelec., Freq. Contr. , p. Submitted, 2015. 

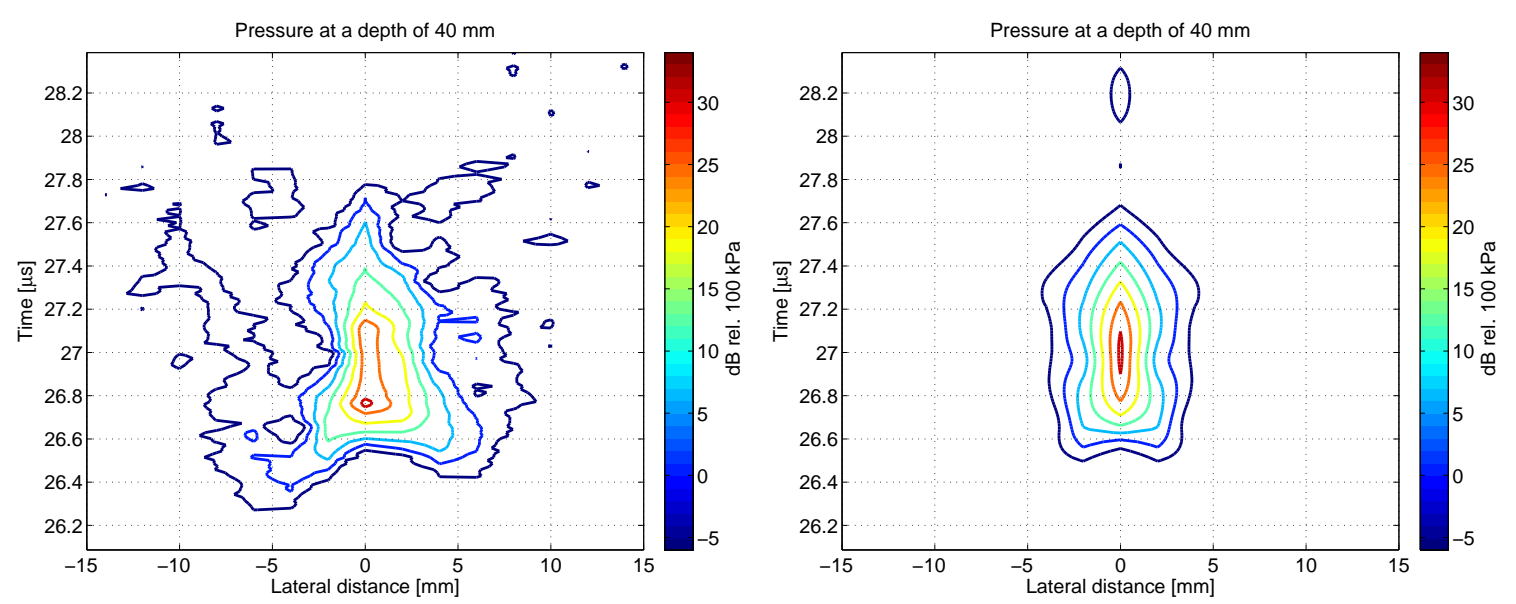

Fig. 10. Pressure distribution of the emitted field for the VFI sequence at a depth of $40 \mathrm{~mm}$. The measured data are shown on the left and the simulated on the right. The scale is shown in $\mathrm{dB}$ relative to $100 \mathrm{kPa}$.

[16] C. A. Villagomez-Hoyos, M. B. Stuart, and J. A. Jensen, "Adaptive multi-lag for synthetic aperture vector flow imaging," in Proc. IEEE Ultrason. Symp., pp. 1722-1725, 2014.

[17] J. A. Jensen, "A multi-threaded version of Field II," in Proc. IEEE Ultrason. Symp., pp. 2229-2232, IEEE, 2014.

[18] G. Pinton and G. Trahey, "Full-wave simulation of finite-amplitude ultrasound in heterogeneous media," in Proc. IEEE Ultrason. Symp., pp. 130-133, 2007.

[19] B. E. Treeby, J. Jaros, A. P. Rendell, and B. T. Cox, "Modeling nonlinear ultrasound propagation in heterogeneous media with power law absorption using a k-space pseudospectral method," J. Acoust. Soc. Am. 131(6), pp. 4324-4336, 2012.

\section{BIBLIOGRAPHIES}

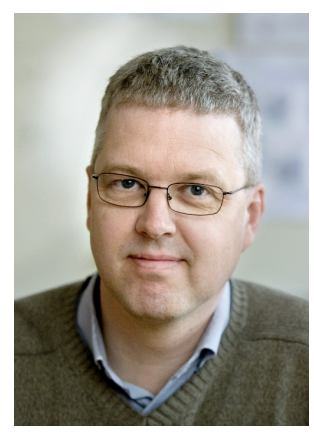

Jørgen Arendt Jensen earned his Master of Science in electrical engineering in 1985 and the Ph.D. degree in 1989, both from the Technical University of Denmark (DTU). He received the Dr.Techn. degree from the university in 1996. He has published more than 400 journal and conference papers on signal processing and medical ultrasound and the book "Estimation of Blood Velocities Using Ultrasound", Cambridge University Press in 1996. He is also developer of the Field II simulation program. He has been a visiting scientist at Duke University, Stanford University, and the University of Illinois at Urbana-Champaign. $\mathrm{He}$ is currently full professor of Biomedical Signal Processing at DTU, Department of Electrical Engineering and head of Center for Fast Ultrasound Imaging since 1998. He has given courses on blood velocity estimation at both Duke University and University of Illinois and teaches medical imaging at the Technical University of Denmark. He has given several short courses on simulation, synthetic aperture imaging, and flow estimation at international scientific conferences. He has received several awards for his research and is an IEEE Fellow. His research is centered around simulation of ultrasound imaging, synthetic aperture imaging, vector blood flow estimation, and construction of ultrasound research systems. 